\title{
Coronavirus Disease 2019 in Patients With Prior Ischemic Stroke
}

Krishna Nalleballe ${ }^{1}$, Suman Siddamreddy ${ }^{2}$, Sen Sheng ${ }^{1}$, Vasuki Dandu ${ }^{3}$, Narenraj Arulprakash ${ }^{1}$, Sukanthi Kovvuru ${ }^{1}$, Mudassar Kamran ${ }^{4}$, Madhu Jasti ${ }^{5}$, Sanjeeva Onteddu ${ }^{1}$

1. Neurology, University of Arkansas for Medical Sciences, Little Rock, USA 2. Internal Medicine, Baptist Health Medical Center, Little Rock, USA 3. Neurology, Baptist Health Medical Center, Little Rock, USA 4. Radiology, University of Arkansas for Medical Sciences, Little Rock, USA 5. Neurology, University of Maryland, Glen Burnie, USA

Corresponding author: Suman Siddamreddy, siddam.suman@gmail.com

\section{Abstract}

Background: It is uncertain if patients with prior ischemic stroke are vulnerable to coronavirus disease 2019 (COVID-19) and its complications.

Methods: We used TriNetX, a global health collaborative clinical research platform with a large global COVID-19 database. COVID-19 infection was identified with a positive lab value for severe acute respiratory syndrome coronavirus 2 (SARS-CoV-2) and related ribonucleic acid (RNA).

Findings: A total of 604,258 patients with history of ischemic stroke were identified, of which 891 patients (study cohort) were diagnosed with COVID-19. A control cohort with 32,136 patients diagnosed with COVID-19 after January $20^{\text {th }} 2020$ without a history of ischemic stroke were identified. A comparison between study cohort and control cohort showed patients with prior history of stroke (study cohort) were older (69.5 vs 47.8 ; $p<0.0001)$ and had more comorbidities contributing to worse clinical outcomes. After propensity matching for demographic variables and comorbidities, only rate of hospitalization (287 vs 231; $\mathrm{p}=0.0035$ ) and need for critical care services ( 85 vs $55 ; \mathrm{p}=0.0082$ ) remained statistically significant while intubation ( 51 vs 43 ; $\mathrm{p}=0.39$ ) and death ( 119 vs $115 ; \mathrm{p}=0.77$ ) showed trends towards worse outcomes but were not statistically significant.

Interpretation: Patients with history of ischemic stroke tend to be significantly older with several comorbid conditions contributing to worse clinical outcomes after COVID-19, which makes them a vulnerable population.

Categories: Internal Medicine, Neurology, Physical Medicine \& Rehabilitation

Keywords: covid 19, stroke

Received 08/27/2020

Review began 08/29/2020 Review ended 09/02/2020 Published 09/03/2020

(c) Copyright 2020

Nalleballe et al. This is an open access article distributed under the terms of the Creative Commons Attribution License CC-BY 4.0., which permits unrestricted use, distribution, and reproduction in any medium, provided the original author and source are credited.

\section{Introduction}

A novel coronavirus, severe acute respiratory syndrome coronavirus 2 (SARS-CoV-2), emerged in December 2019 and soon led to a worldwide pandemic, coronavirus disease 2019 (COVID-19) [1]. COVID-19 has been shown to cause several neurological manifestations including ischemic strokes [2-4]. Most of the predictions about risk factors, clinical characteristics, and pathophysiology are based on our experience with previous coronaviruses, i.e. severe acute respiratory syndrome coronavirus (SARS-CoV) and Middle East respiratory syndrome coronavirus (MERS-CoV). Stroke is one of the chronic diseases with very high morbidity and mortality causing a huge financial burden to the healthcare economy [5]. While there are many studies looking at risk factors for severe COVID-19 and its complications, 'history of ischemic stroke' as a risk factor contributing to the severity of COVID-19 has not been studied in a large population [6]. The purpose of this study is to explore if patients with prior ischemic stroke are a vulnerable population for worse clinical outcomes in patients with COVID-19.

\section{Materials And Methods \\ Data source}

De-identified patient information data for COVID-19 and history of ischemic stroke were extracted from TriNetX (www.trinetx.com), which is a global health collaborative clinical research platform collecting realtime electronic medical records data from a network of health care organizations (HCOs). "COVID-19 Research Network" in TriNetX is a large COVID-19 database, which is also being used by the Food \& Drug Administration (FDA) Sentinel Operations Center at the Harvard Pilgrim Health Care Institute to monitor priority drugs used for the care of the hospitalized COVID-19 patients [7].

Queries were made through TriNetX using an internet browser and accessing its real-time features. TriNetX does not allow data downloads or individual patient data for review. However, the TriNetX platform allows 
analysis in the form of queries. The TriNetX platform has previously been described in detail in several similar studies on COVID-19 that used this platform [3,8,9,10,11]. At University of Arkansas for Medical Sciences, the data from TriNetX is managed by the Arkansas Clinical Data Repository (AR-CDR) and maintained by the Department of Biomedical Informatics.

\section{Study protocol}

The University of Arkansas Institutional Review Board (IRB) deemed this study to be 'not human subject research' (global de-identified COVID-19 Research Network data designated for research use), and gave it an IRB exempt status.

Data were accessed on July 20, 2020 on TriNetX COVID-19 Research Network. Using ICD-10 codes, individuals $\geqslant 18$ years of age with a pre-existing ischemic stroke (I63) were identified. Among them, we then examined patients with a confirmed lab-positive reverse transcription polymerase chain reaction (RTPCR) for COVID-19 (study cohort). For comparison, a control cohort with 32,136 patients diagnosed with COVID-19 after January 20, 2020 without a history of ischemic stroke were identified. Between the two cohorts, hospitalizations, critical care services, intubation, and mortality data within one month from COVID-19 diagnosis were studied. Additionally, baseline demographics data and clinical outcomes in these cohorts were examined. Statistical analysis was performed using the TriNetX analytics function. Descriptive statistics are reported as number of observations, percentage, or mean \pm standard deviation as applicable. One-to-one propensity score matching was done for baseline characteristics and comorbid conditions. The $\mathrm{p}$-value for variables is generated from a t-test. For the outcomes however, a z-test is used for the p-value.

\section{Results}

There were 604,258 patients with prior history of ischemic stroke, of which 891 (0.15\%) patients developed COVID-19 (study cohort). Among them 451 (50.6\%) were females; 409 (45.9\%) Caucasians, 278 (31.2\%) African Americans and 32 (3.6\%) Asians. Ethnicity and comorbid conditions are detailed in Table 1 . The control cohort included 32,136 patients. Baseline characteristics, gender, race, comorbid conditions of this cohort are also detailed in Table 1. 


\section{Cureus}

\begin{tabular}{|c|c|c|c|}
\hline & Study Cohort & Control Cohort & p-value \\
\hline Number of patients & 891 & 32,136 & NA \\
\hline Age (years) & $69.5 \pm 14.5$ & $47.8 \pm 18.9$ & $<0.0001$ \\
\hline Woman & $451(50.6 \%)$ & $16,956(52.7 \%)$ & 0.21 \\
\hline \multicolumn{4}{|l|}{ Race } \\
\hline White & $409(45.9 \%)$ & $12,220(38.0 \%)$ & $<0.0001$ \\
\hline African American & $278(31.2 \%)$ & 6,177(19.2\%) & $<0.0001$ \\
\hline Asian & $32(3.6 \%)$ & $641(1.9 \%)$ & 0.0009 \\
\hline Hispanic (ethnicity) & $68(7.6 \%)$ & $4,564(14.2 \%)$ & $<0.0001$ \\
\hline \multicolumn{4}{|l|}{ Co morbid conditions } \\
\hline Hypertension & $734(82.4 \%)$ & 7,489(23.3\%) & $<0.0001$ \\
\hline Diabetes mellitus & $426(47.8 \%)$ & $3,986(12.4 \%)$ & $<0.0001$ \\
\hline Ischemic heart disease & $394(44.2 \%)$ & $2,102(6.5 \%)$ & $<0.0001$ \\
\hline Chronic kidney disease & 297(33.3\%) & $1,617(5.0 \%)$ & $<0.0001$ \\
\hline Heart failure & $303(34.0 \%)$ & $1,453(4.5 \%)$ & $<0.0001$ \\
\hline Atrial fibrillation \& flutter & $241(27.1 \%)$ & $1,136(3.6 \%)$ & $<0.0001$ \\
\hline Obstructive pulmonary disease & 188(21.1\%) & $1,125(3.5 \%)$ & $<0.0001$ \\
\hline Overweight \& obesity & $297(33.3 \%)$ & 4,603(14.3\%) & $<0.0001$ \\
\hline Nicotine dependence & 169(18.9\%) & $2,039(6.3 \%)$ & $<0.0001$ \\
\hline Liver Disease & $79(8.9 \%)$ & $1,251(3.9 \%)$ & $<0.0001$ \\
\hline \multicolumn{4}{|l|}{ Clinical outcome } \\
\hline Hospitalization & $289(32.4 \%)$ & $3,746(11.7 \%)$ & $<0.0001$ \\
\hline Critical care services & $85(9.5 \%)$ & $711(2.2 \%)$ & $<0.0001$ \\
\hline Intubation & $51(5.7 \%)$ & $642(1.9 \%)$ & $<0.0001$ \\
\hline Death & $119(13.4 \%)$ & $1,143(3.6 \%)$ & $<0.0001$ \\
\hline
\end{tabular}

\section{TABLE 1: Table 1. Characteristics and outcomes of patients with COVID-19}

Baseline demographics, comorbidities and outcomes for Study and Control cohort.

Study Cohort: Patients with COVID-19 and history of ischemic stroke.

Control Cohort: Patients with COVID-19 without history of ischemic stroke.

[NA-Not applicable]

Among these two cohorts, patients in the study cohort were older ( 69.5 years vs 47.8 years; $\mathrm{p}<0.0001$ ), had significantly higher prevalence of hypertension, diabetes mellitus, ischemic heart disease, chronic kidney disease, heart failure, atrial fibrillation and flutter, obstructive pulmonary disease, overweight and obesity, liver disease, and nicotine dependence ( $\mathrm{p}<0.0001$, Table 1$)$. When comparing for COVID-19 outcomes, unmatched analysis showed statistically higher rates of hospitalization ( $32.4 \%$ vs $11.7 \%$, $\mathrm{p}<0.0001$ ), need for critical care services $(9.5 \%$ vs $2.2 \%$, $\mathrm{p}<0.0001)$, intubation $(5.7 \%$ vs $1.9 \%$, $\mathrm{p}<0.0001)$ and death $(13.4 \%$ vs $3.6 \%, \mathrm{p}<0.0001$ ) (Table 1 ) among the study cohort. After propensity score matching for demographics and comorbidities, each group had 888 patients. Rate of hospitalization ( $32.3 \%$ vs $26.0 \%, \mathrm{p}=0.0035)$ and need for critical care services $(9.5 \%$ vs $6.2 \%, \mathrm{p}=0.0082)$ was higher in the study cohort and was statistically significant. While intubation ( $5.7 \%$ vs $4.8 \%, \mathrm{p}=0.39$ ) and death $(13.4 \%$ vs $12.9 \%, \mathrm{p}=0.77)$ showed trends towards worse outcomes they were not statistically significant (Table 2). 


\section{Cureus}

\begin{tabular}{|c|c|c|c|}
\hline & Study Cohort & Control Cohort & p-value \\
\hline Number of patients & 888 & 888 & NA \\
\hline Age (years) & $69.5 \pm 14.5$ & $70 \pm 14.9$ & 0.48 \\
\hline Woman & $450(50.7 \%)$ & $433(48.8 \%)$ & 0.42 \\
\hline \multicolumn{4}{|l|}{ Race } \\
\hline White & 409(46.1\%) & $422(47.5 \%)$ & 0.54 \\
\hline African American & $277(31.2 \%)$ & $258(29.1 \%)$ & 0.33 \\
\hline Asian & $30(3.4 \%)$ & $31(3.5 \%)$ & 0.89 \\
\hline Hispanic (ethnicity) & $68(7.7 \%)$ & $67(7.6 \%)$ & 0.93 \\
\hline \multicolumn{4}{|l|}{ Co morbid conditions } \\
\hline Hypertension & $731(82.3 \%)$ & $751(84.6 \%)$ & 0.2 \\
\hline Diabetes mellitus & $424(47.8 \%)$ & $423(47.6 \%)$ & 0.96 \\
\hline Ischemic heart disease & $391(44.0 \%)$ & $372(41.9 \%)$ & 0.36 \\
\hline Chronic kidney disease & $294(33.1 \%)$ & $282(31.8 \%)$ & 0.54 \\
\hline Heart failure & $300(33.8 \%)$ & 278(31.3\%) & 0.26 \\
\hline Atrial fibrillation \& flutter & 239(26.9\%) & $242(27.2 \%)$ & 0.87 \\
\hline Obstructive pulmonary disease & $187(21.1 \%)$ & $181(20.4 \%)$ & 0.73 \\
\hline Overweight \& obesity & $295(33.2 \%)$ & 298(33.6\%) & 0.88 \\
\hline Nicotine dependence & 168(18.9\%) & 144(16.2\%) & 0.13 \\
\hline Liver Disease & $79(8.9 \%)$ & $84(9.5 \%)$ & 0.68 \\
\hline \multicolumn{4}{|l|}{ Cilinical outcome } \\
\hline Hospitalization & 287(32.3\%) & $231(26.0 \%)$ & 0.0035 \\
\hline Critical care services & $85(9.5 \%)$ & $55(6.2 \%)$ & 0.0082 \\
\hline Intubation & $51(5.7 \%)$ & $43(4.8 \%)$ & 0.39 \\
\hline Death & 119(13.4\%) & $115(12.9 \%)$ & 0.77 \\
\hline Composite end point & $198(22.3 \%)$ & $161(18.1 \%)$ & 0.0288 \\
\hline
\end{tabular}

\section{TABLE 2: Characteristics and outcomes of patients with COVID-19 after propensity matching}

Baseline demographics, comorbidities and outcomes for Study and Control cohort.

Study Cohort: Patients with COVID-19 and history of ischemic stroke.

Control Cohort: Patients with COVID-19 without history of ischemic stroke.

Composite end point includes hospitalizations, critical care services, intubations and death.

[NA-Not applicable]

A composite endpoint which included hospitalizations, critical care services, intubations and death was significant in patients with prior history of ischemic strokes $(22.3 \%$ vs $18.1 \%, \mathrm{p}=0.0288)$.

\section{Discussion}

In this study, we explored if prior ischemic stroke is an independent risk factor for worse clinical outcomes in patients with COVID-19. Identifying vulnerable populations may allow patients and healthcare professionals to take extra precautions to prevent the occurrence of disease as well as its complications, 
A history of prior stroke has been shown to be associated with worse outcomes in SARS-CoV and MERS-CoV $[12,13]$. Even in other respiratory illnesses, like community-acquired pneumonia, stroke has been associated with higher mortality [14]. A recent meta-analysis showed that COVID-19 patients with underlying hypertension, cardiovascular disease, and previous respiratory diseases are at higher risk of having severe disease when compared to patients without those comorbidities [15]. Patients with previous cardiovascular disease have increased risk of death with COVID-19 infection [16]. Pooled analysis of six studies done by Aggarwal et al. showed that there is a 2.5 -fold increase in odds of severe COVID-19 infection in patients with previous strokes, but no significant association with mortality; however, the analysis had few studies with small sample size [17]. Similarly, a study by Qin et al. showed that after propensity score matching patients with history of stroke had higher risk of severe events including mortality, critical care admission, intubations, and lower rates of discharge [6]. Patients with history of stroke tend to be older with several comorbidities which likely resulted in severe COVID-19 complications. After propensity matching, we noticed that history of ischemic stroke does not seem to be an independent risk factor for intubations and deaths.

There are a few limitations of this study, some of them are inherent to large databases. First, findings are based on reported data, and accuracy of reported data could not be verified. Second, we could not assess the duration and severity of prior ischemic stroke.

\section{Conclusions}

Patients with history of prior ischemic stroke tend to be older with several comorbid conditions, which contribute to severe COVID-19 complications and make them a vulnerable population. Future large prospective studies may provide additional insights into this relationship.

\section{Additional Information}

\section{Disclosures}

Human subjects: Consent was obtained by all participants in this study. IRB at University of Arkansas for Medical Sciences issued approval NA. The University of Arkansas Institutional Review Board (IRB) deemed this study to be 'not human subject research' (global de-identified COVID-19 Research Network data designated for research use), and gave it an IRB exempt status. . Animal subjects: All authors have confirmed that this study did not involve animal subjects or tissue. Conflicts of interest: In compliance with the ICMJE uniform disclosure form, all authors declare the following: Payment/services info: All authors have declared that no financial support was received from any organization for the submitted work. Financial relationships: All authors have declared that they have no financial relationships at present or within the previous three years with any organizations that might have an interest in the submitted work. Other relationships: All authors have declared that there are no other relationships or activities that could appear to have influenced the submitted work.

\section{References}

1. Coronavirus Disease (COVID-19) Weekly Epidemiological Update and Weekly Operational Update . (2020). Accessed: August 1, 2020: https://www.who.int/emergencies/diseases/novel-coronavirus-2019/situationreports.

2. Avula A, Nalleballe K, Narula N, et al.: COVID-19 presenting as stroke. Brain Behav Immun. 2020, 10:1016. 10.1016/j.bbi.2020.04.077

3. Onteddu SR, Nalleballe K, Sharma R, Brown AT: Underutilization of healthcare for strokes during the COVID-19 outbreak. Int J Stroke. 2020, 174749302093436. 10.1177/1747493020934362

4. Jasti M, Nalleballe K, Dandu V, Onteddu S: A review of pathophysiology and neuropsychiatric manifestations of COVID-19. J Neurol. 2020, 10.1007/s00415-020-09950-w

5. Virani S, Alonso A, Benjamin E, et al.: Heart disease and stroke statistics-2020 update: a report from the American Heart Association. Circulation. 2020, 141:139-151. 10.1161/CIR.0000000000000757

6. Qin C, Zhou L, Hu Z, et al.: Clinical characteristics and outcomes of COVID-19 patients with a history of stroke in Wuhan, China. Stroke. 2020, 51:2219-2223. 10.1161/STROKEAHA.120.030365

7. TriNetX. (2020). http://www.trinetx.com/coronavirus.

8. Nalleballe K, Reddy Onteddu S, Sharma R, et al.: Spectrum of neuropsychiatric manifestations in COVID-19. Brain Behav Immun. 2020,

9. Stapff M, Hilderbrand S: Firstline treatment of essential hypertension: a real-world analysis across four antihypertensive treatment classes. J Clin Hypertens. 2019, 21:627-634.

10. Ranabothu S, Onteddu S, Nalleballe K, Dandu V, Veerapaneni K, Veerapandiyan A: Spectrum of COVID-19 in children. Acta Paediatr. 2020, 10.1111/apa.15412

11. Turk MA, Landes SD, Formica MK, Goss KD: Intellectual and developmental disability and COVID-19 casefatality trends: TriNetX analysis. Disabil Health J. 2020, 100942-10. 10.1016/j.dhjo.2020.100942

12. Chen C, Lee C, Liu C, Wang J, Wang L, Perng R: Clinical features and outcomes of severe acute respiratory syndrome and predictive factors for acute respiratory distress syndrome. J Chin Med Assoc. 2005, 68:4-10. 10.1016/S1726-4901(09)70124-8

13. Lee SY, Khang YH, Lim HK: Impact of the 2015 Middle East respiratory syndrome outbreak on emergency 


\section{Cureus}

care utilization and mortality in South Korea. Yonsei Med J. 2019, 60:796-803. 10.3349/ymj.2019.60.8.796

14. Mandal P, Chalmers JD, Choudhury G, Akram AR, Hill AT: Vascular complications are associated with poor outcome in community-acquired pneumonia. QJM. 2011, 104:489-495. 10.1093/qjmed/hcq247

15. Yang J, Zheng Y, Gou X, et al.: Prevalence of comorbidities and its effects in patients infected with SARSCoV- 2: a systematic review and meta-analysis. Int J Infect Dis. 2020, 94:91-95. 10.1016/j.ijid.2020.03.017

16. Ruan Q, Yang K, Wang W, Jiang L, Song J: Clinical predictors of mortality due to COVID-19 based on an analysis of data of 150 patients from Wuhan, China. Intens Care Med. 2020, 46:846-848. 10.1007/s00134020-05991-x

17. Aggarwal G, Lippi G, Michael Henry B: Cerebrovascular disease is associated with an increased disease severity in patients with coronavirus disease 2019 (COVID-19): a pooled analysis of published literature. Int J Stroke. 2020, 15:385-389. 